
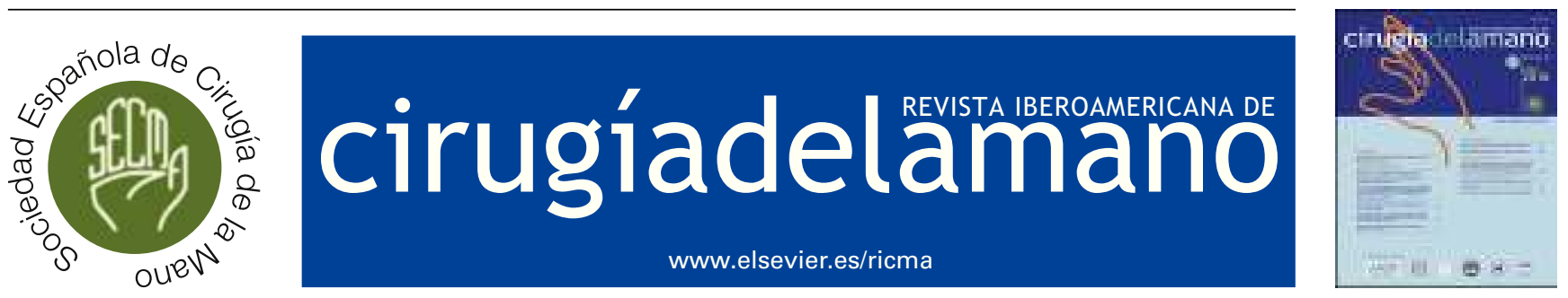

ARTÍCULO ESPECIAL

\title{
Cómo escribir un artículo original en Cirugía de la Mano
}

\author{
R.S. Rosales ${ }^{a, *}$, R. García Gutiérrez ${ }^{\mathrm{b}}$, Y. Martín Hidalgo ${ }^{\mathrm{b}}$, \\ L. Reboso Morales ${ }^{\mathrm{b}}$ e I. Atroshi ${ }^{\mathrm{c}, \mathrm{d}}$ \\ a Unidad de Cirugía de la Mano y Microcirugía, GECOT, La Laguna, Tenerife, España

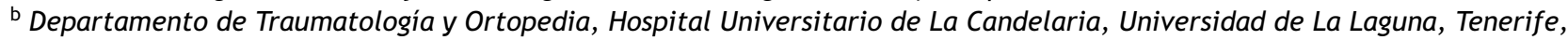 \\ España \\ ' Departments of Clinical Sciences, Lund University, Lund, Suecia \\ d Orthopedics Hässleholm-Kristianstad, Hässleholm Hospital, Hässleholm, Suecia
}

Recibido el 14 de febrero de 2016; aceptado el 19 de febrero de 2016

Disponible en Internet el 1 de abril de 2016

\section{PALABRAS CLAVE \\ Escritura científica; Publicación \\ científica; \\ Investigación clínica}

\section{KEYWORDS}

Scientific writing; Scientific journal; Clinical research

\begin{abstract}
Resumen Considerar la calidad de la «escritura científica» a la hora de difundir nuevas ideas, resultados de tratamientos o nuevos descubrimientos, es un paso fundamental en la publicación científica. Pocos cirujanos de mano han recibido formación específica en este campo durante su residencia o entrenamiento. El propósito del presente artículo es presentar los principios fundamentales de la escritura científica a la hora de desarrollar un artículo original en investigación, fundamentalmente en la clínica, en el campo de la Cirugía de la Mano.

( $) 2016$ SECMA. Publicado por Elsevier España, S.L.U. Este es un artículo Open Access bajo la licencia CC BY-NC-ND (http://creativecommons.org/licenses/by-nc-nd/4.0/).
\end{abstract}

\section{Writing an original scientific article on Hand Surgery}

Abstract The quality of scientific writing needs to be taken into consideration as a first step in the dissemination of new ideas, treatment outcomes, and new discoveries. Few hand surgeons have received specific training on this issue during their residency or training courses. The purpose of this paper is to present the basic principles of writing scientific articles, especially in clinical research and in hand surgery.

(c) 2016 SECMA. Published by Elsevier España, S.L.U. This is an open access article under the CC BY-NC-ND license (http://creativecommons.org/licenses/by-nc-nd/4.0/).
Desde su origen en 1665, los trabajos científicos han sufrido cambios en su presentación y estilo. En los 2 primeros siglos de historia de la escritura científica, el formato y el estilo no

\footnotetext{
* Autor para correspondencia.

Correo electrónico: titorosales@telefonica.net (R.S. Rosales).
}

estaban estandarizados. Así, el formato de «carta» al editor coexistía con los trabajos que reportaban hallazgos experimentales. Las cartas eran generalmente firmadas por un solo autor, escritas en un formato «educado» y abordaban diferentes tópicos al mismo tiempo ${ }^{1}$. Los trabajos experimentales eran puramente descriptivos y los eventos eran expuestos de forma cronológica, para después evolucionar a 
una estructura en la que el método y los resultados estaban mejor descritos e interpretados, mientras que las clásicas cartas y los trabajos en formato carta desaparecían ${ }^{2}$. La descripción del «método» fue incrementándose en los trabajos científicos durante la segunda mitad del siglo $\mathrm{XIX}^{3}$, apareciendo una nueva organización de los manuscritos científicos que fue conocida como «teoría-experimento-discusión» ${ }^{4}$.

A principios del siglo $\mathrm{xx}$, las normas editoriales, parecidas a las contemporáneas, comenzaron a estandarizarse, con un claro detrimento en el uso del estilo literario en las publicaciones científicas. Gradualmente, en el curso del siglo $x x$, el formato introducción, método, resultados y discusión (IMRAD) fue adoptado ${ }^{5}$. A partir de aquí, la frecuencia de artículos científicos usando el formato IMRAD se ha ido incrementando en el tiempo. En 1935, no existía ningún artículo con estructura IMRAD; en 1950, la proporción de artículos con formato IMRAD superaba el $10 \%$ en todas las revistas importantes como British Medical Jornal (BMJ), The Lancet, The Journal of the American Medical Association (JAMA) y New England Journal of Medicine. Entre 1955 y 1975 la frecuencia de artículos con formato IMRAD se cuadruplicó, y en 1970 , el $80 \%$ de los artículos tenía ya este formato ${ }^{6}$.

En algún momento, la mayoría de los profesionales médicos en general y los cirujanos de mano en particular, se ven envueltos en la necesidad de escribir manuscritos científicos durante el desarrollo de su actividad profesional. Sin embargo, la residencia o los programas de formación más especializados, como los fellowship, no ofrecen formación sobre la escritura, estructura y formato que deben poseer los artículos científicos. Normalmente, los autores aprenden el proceso de la escritura científica de la lectura de otros trabajos publicados, con muy poca ayuda o guía por parte de sus jefes o mentores. A pesar de que la lectura y crítica de artículos científicos puede ser una herramienta valiosa para llegar a ser un buen escritor científico, la buena escritura de artículos científicos requiere de un conocimiento básico de la estructura y formato de los manuscritos científicos y del entendimiento de los mismos ${ }^{7-10}$.

El propósito del presente trabajo ha sido definir qué recomendaciones se precisan a la hora de escribir cada una de los apartados de un trabajo científico, fundamentalmente en el área de la investigación clínica, usando el formato IMRAD, aceptado en las normas editoriales de la REVISTA IBEROAMERICANA DE CIRUGÍA DE LA MANO (RICMA). A pesar de que el presente trabajo se dedica en principio a los lectores y futuros autores de la revista RICMA, creemos que este artículo será útil y relevante para la escritura en cualquier revista médica.

\section{Antes de empezar}

Antes de empezar a escribir, sería conveniente seleccionar el journal o revista que mejor se adapte al trabajo e ideas que quiera difundir. En nuestro caso, la RICMA tiene cada vez más una mayor influencia, no solo en el mundo iberoamericano, sino que también se han confirmado numerosas descargas desde otros países como EE. UU. Es importante leer claramente las normas de la revista antes de empezar a escribir (http:/ / www.elsevier.es/es-revista-revista-iberoamericanacirugia-mano-134-normas-publicacion). Es conveniente leer los números más recientes para tener una primera impresión del estilo recomendado y usado en la revista (muchos trabajos son rechazados porque no siguen las recomendaciones de las normas editoriales de autor expuestas por el editor de la revista científica) ${ }^{11}$.

El proceso de escritura de un artículo original en Cirugía de la Mano es similar al de «contar una historia». Al igual que una historia bien escrita, un trabajo científico tendrá un comienzo (la introducción), una mitad (el método), un final (los resultados) y una «moraleja» (la discusión). Se discute hoy en día si la escritura de un trabajo de investigación clínica debería empezar por el título seguido del abstract o resumen. Autores como Kotsis y Chung ${ }^{7-9}$ y Manske ${ }^{10}$ (antiguo editor del Journal of Hand Surgery American [JHSA]) recomiendan escribir al final del artículo las partes más importantes y las más leídas como son el «abstract» y el «título». En la «introducción» se explica «el porqué» de nuestro trabajo; en el «método» se expone «el cómo»se hizo nuestro trabajo; en la sección «resultados» se expone «lo descubierto» con nuestra investigación, y finalmente la «discusión» o «moraleja» constituye «la interpretación»de nuestros resultados ${ }^{10}$. Aunque todas las secciones IMRAD son importantes, quizá «la introducción» y «el método» ${ }^{12}$ constituyen las de mayor atención.

\section{Escribir la introducción}

En la introducción, denominada como background en revistas de lengua anglosajona como el JAMA, The Lancet, Plastic and Reconstructive Surgery, etc., es donde los autores deben exponer claramente «el porqué» de su trabajo, qué tiene de diferente o innovador, cuál es la importancia u originalidad de su investigación y cuál es su relevancia clínica.

La introducción debe comenzar exponiendo cuál es el conocimiento científico actual sobre el tema objeto del estudio (qué se conoce), cuál es el vacío en el conocimiento (qué no se conoce), generar la pregunta de investigación e hipótesis del trabajo (sintetiza la pregunta científica a la que se pretende dar respuesta con el trabajo de investigación), para finalizar con la parte fundamental que es el objetivo o propósito del estudio de investigación (qué se quiere conocer). La pregunta de investigación representa la incertidumbre del investigador sobre algún fenómeno que ocurre en la población y debe ser: importante (por la gravedad y/o frecuencia), interesante (a la luz de los conocimientos actuales), que tenga respuesta (con los medios disponibles) y realizable (en un tiempo concreto) ${ }^{12-15}$. Por tanto, la introducción es, junto con el método, una de las piedras angulares de la que dependerá la aceptación o no de un trabajo de investigación en cualquier journal.

La introducción es una de las partes más difíciles de escribir del manuscrito científico. A pesar de que algunos autores como Kotsis y Chung $^{7}$ en sus recomendaciones para escribir artículos para el JHSA recomiendan escribir la introducción en «tiempo presente», nuestro equipo ${ }^{13}$ y la mayoría de las revistas científicas ${ }^{16}$ recomiendan escribir esta sección del manuscrito en «tiempo pasado», sobre todo cuando hablamos de lo ya publicado por otros. Una introducción competente debe incluir: 1) la significación o importancia del tópico, 2) la información sobre el gap en la literatura científica sobre dicho tópico, 3) una precisa revisión bibliográfica que sustente la pregunta de investigación, y 4) el desarrollo de los propósitos/objetivos e hipótesis. 
Errores en la escritura de la introducción suelen ser: 1) una excesiva revisión bibliográfica, demasiadas citas y una larga introducción, que recuerdan más a la escritura de un capítulo de un libro o tesis doctoral que a un trabajo científico ${ }^{16}$. Por ejemplo, si vamos a introducir un trabajo clínico sobre la prevalencia de la enfermedad de Dupuytren (ED) en la población general, no será necesario escribir 2 o 3 párrafos hablando sobre generalidades de la ED; debemos comenzar la introducción escribiendo sobre lo más relevante que se haya publicado de la prevalencia de la ED. 2) Una revisión bibliográfica incompleta, imprecisa y fuera de actualidad es una de las causas comunes de rechazo de trabajos originales de investigación ${ }^{7}$. 3) Probablemente el mayor error de la introducción y de todo el manuscrito sea la ausencia de una declaración precisa del propósito del trabajo de investigación. Ello es debido a que no existe un propósito específico como tal, ya que la mayoría de los artículos publicados en las revistas científicas de Cirugía de la Mano son serie de casos (nivel 4 de evidencia científica) ${ }^{17,18}$, estudios que tienen un diseño por definición descriptivo, y por tanto retrospectivo, y el proyecto no ha comenzado con la pregunta de investigación. Frecuentemente, los autores revisan todos los casos sobre una patología determinada y, una vez recolectados los datos, es cuando se plantea el objeto de la investigación. En ciertas ocasiones este orden inverso en la metodología de la investigación puede ser efectivo, pero los autores deben identificar la pregunta de investigación y el propósito del trabajo, o en caso contrario los revisores y lectores rápidamente captaran la impresión de que los autores fueron recolectando primero los datos, los reunieron y luego los pusieron juntos en forma de manuscrito. Ese tipo de manuscrito suele carecer de valor. Para evitar ese error hay que evitar frases como: «Nosotros decidimos revisar...», «En este trabajo revisamos nuestra experiencia en...», «Presentamos una revisión de...», y sobre todo nunca usar las palabras retrospectivo o prospectivo, ya que no dicen nada sobre el diseño clínico y, por ende, del nivel de evidencia científica del trabajo, como veremos en el apartado del «método». Una declaración apropiada del propósito de un trabajo científico suele comenzar con frases como: «El propósito del presente trabajo fue...».

En resumen, una buena «introducción» comenzaría con un primer párrafo que mencione las referencias más importantes a la hora de establecer el problema de investigación en cuestión. Un segundo párrafo puede elaborar o exponer la magnitud del problema de investigación y aquellas preguntas sin resolver. El párrafo final expone la razón e importancia del trabajo de investigación, finalizando con el propósito y la hipótesis de investigación.

\section{Escribir el método}

El método es la parte más importante, junto con la introducción, de un trabajo de investigación ${ }^{12}$. A diferencia de la introducción, nunca se deben tener límites a la hora de escribir en esta sección del manuscrito. La sección «método» deber ser escrita lo suficientemente detallada de manera que otro investigador pueda reproducir o duplicar su investigación. El «método» es la sección del manuscrito que causa más rechazos en la publicación científica. En esta sección, los autores deben explicar todo lo que hicieron y cómo lo hicieron para contestar a la pregunta de investigación que fue expuesta en la introducción. El «método» siempre se escribe en «tiempo pasado».

La sección «método» debe incluir los siguientes subapartados: población estudio, diseño clínico, instrumentos y medidas, y análisis estadístico.

\section{Población estudio}

Claramente, los autores tienen que establecer lo que se denomina en investigación clínica: «definición de caso» o conjunto de criterios estándares que permiten decidir si una persona tiene una particular enfermedad o estado relacionado con la salud. Los criterios para la «definición de caso» pueden ser clínicos o de pruebas complementarias. Se clasifican en criterios de inclusión y de exclusión. Mediante el uso de criterios estándares, los autores pueden asegurarse de que cada caso fue diagnosticado de la misma manera con independencia de cuándo, dónde o quién realizó la identificación de los casos. Los problemas que nos encontramos a la hora de categorizar casos se denominan «sesgo de información». Dos aspectos importantes del sesgo de información son: la fiabilidad (error intraobservador y error interobservadores) y la validez (sensibilidad, especificidad y precisión) de los criterios utilizados para definir los $\operatorname{casos}^{12}$.

La definición de la población estudio debe comenzar con los criterios de inclusión y exclusión empleados para definir cada caso. Debemos incluir citas bibliográficas de cada criterio empleado que nos aporten luz sobre la fiabilidad y validez de los mismos. A continuación, se debería continuar con el «reclutamiento» de los pacientes aportando información sobre quién (entre paréntesis las iniciales en mayúscula del autor encargado de dicho proceso) constituyó la población estudio, cómo y de dónde (e.g.: de lista de espera, a través de encuesta de salud, de los pacientes que acudían a urgencias del hospital «X» con una población de referencia de $« X »$ habitantes, etc.). Para este apartado, se recomienda incluir un diagrama de flujo de pacientes hasta su inclusión en la población estudio que variará dependiendo el diseño clínico empleado para contestar a la pregunta de investigación. En el siguiente apartado veremos ejemplos de un flujo de pacientes en una población estudio en un diseño de cohortes (fig. 1), en un ensayo clínico aleatorizado (ECA) (fig. 2) y en un metaanálisis (MA) o revisión sistemática (RS) (fig. 3).

Toda la información demográfica (edad, sexo, número de sujetos en cada grupo, tiempo de seguimiento, características específicas de grupos comparados, como medidas base de discapacidad, calidad de vida, etc.) que describen a los sujetos de la población estudio, debe ser incluida en esta sección. Mejor mediante una tabla demográfica, teniendo en cuenta el evitar en lo posible los porcentajes, y usando mejor fracciones (e.g.: 20/40 en vez del 50\%). Es un error muy común empezar la sección «resultados» con los datos demográficos de la población estudio. Esa sección del trabajo científico debe ser reservada a los datos obtenidos con las mediciones resultados u outcomes. Debemos recordar que los datos demográficos no se incluyen en la sección «resultados» ${ }^{10}$. La tabla demográfica se complementa en el texto con un pequeño párrafo que resume la población estudio definida. A continuación presentamos un 


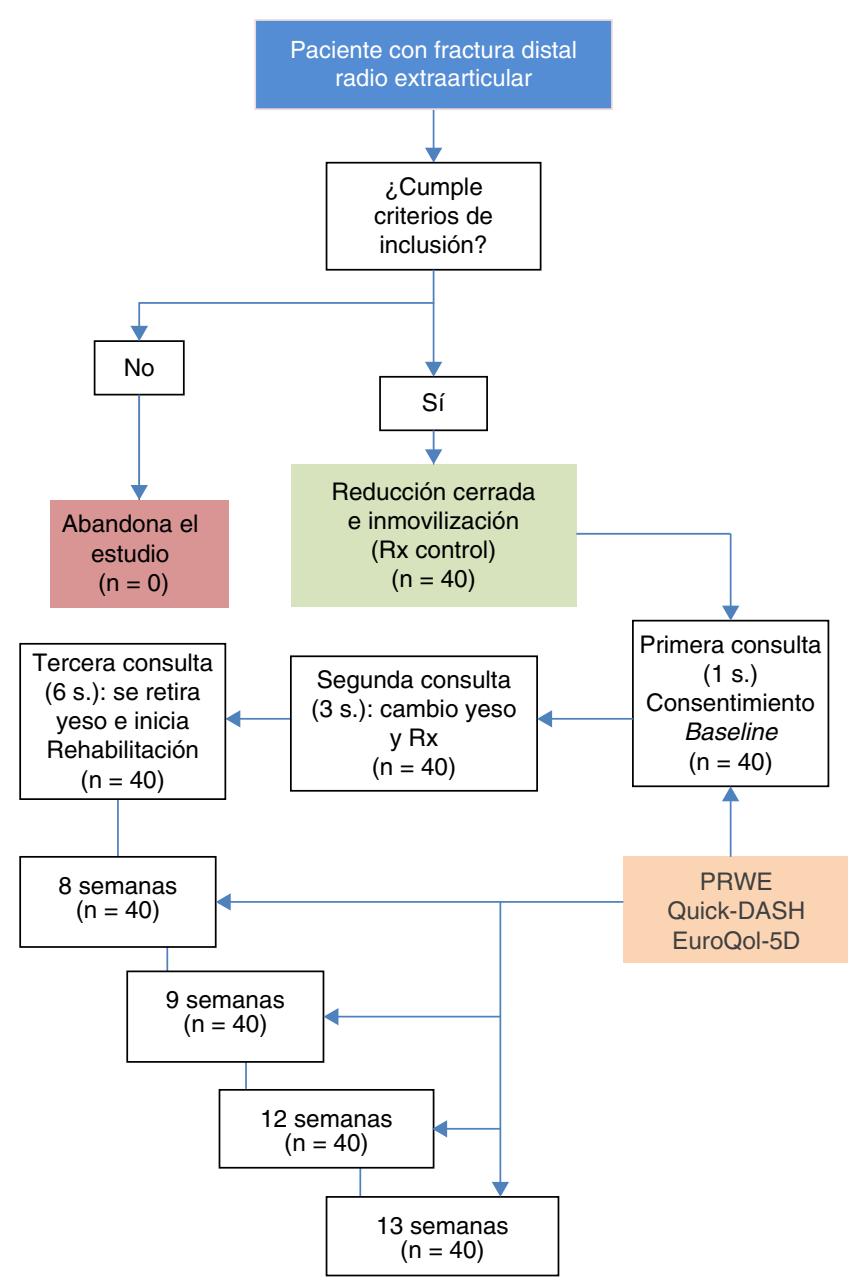

Figura 1 Diagrama de flujo en un diseño clínico «cohortes clásico» o longitudinal prospectivo con medidas antes y después del tratamiento.

Fuente: adaptado de García Gutierrez ${ }^{15}$.

ejemplo de un diseño de cohortes clásico: «Una cohorte de 40 pacientes consecutivos (31 mujeres), con una edad media de 58 años con fractura distal de radio extra articular, tratados mediante reducción cerrada e inmovilización constituyeron la población del estudio (fig. 1). No hubo pérdidas de seguimiento» ${ }^{15}$.

La subsección «población estudio» se puede empezar o terminar, según los requerimientos de cada revista científica, con un pequeño párrafo que hace alusión a la preservación de los derechos en investigación realizada en humanos $y$, en caso de ECA, aportar el número de aceptación para el ensayo dado por el Comité Ético de la institución responsable. En las normas editoriales de la RICMA, carta de remisión apartado 5 , se describe claramente este punto, que debe ser aceptado y firmado por los autores. Además, académicamente se debería incluir un pequeño párrafo como el que sigue a continuación en el texto del manuscrito dentro de esta subsección: «Todos los procedimientos relacionados en este estudio donde intervinieron pacientes estuvieron en consonancia con los estándares éticos del Comité de Investigación Institucional y Nacional del Hospital Universitario Nuestra Señora de La Candelaria, y con la declaración de Helsinki de 1964 y sus enmiendas. Se obtuvo el consentimiento informado de todos los pacientes participantes en el estudio».

\section{Diseño clínico}

Describir claramente el diseño clínico empleado. No usar las palabras «retrospectivo» o «prospectivo» pues no aportan información sobre el diseño clínico y, por ende, del nivel de evidencia científica del trabajo de investigación. Por ejemplo: un estudio de casos-control, un estudio de cohortes clásico y un estudio de corte transverso son retrospectivos, pero con diseños clínicos distintos y un nivel de evidencia diferente ${ }^{12,13}$.

\section{Estudios observacionales descriptivos}

Constituyen el nivel más bajo de la evidencia científica. En este nivel se incluye a la mayoría de trabajos que se publican hoy en día o que se presentan a congresos de sociedades científicas. Todos tienen un denominador común: suelen ser retrospectivos y solo se incluye una medición en el tiempo. Se pueden clasificar en: estudios que recogen información individual (informe de un caso, serie de casos, vigilancia epidemiológica y estudios transversales), que son los más extendidos en nuestra especialidad, o de grupo (estudios ecológicos), de muy poco uso en Cirugía de la Mano. En este tipo de estudios, la opinión del experto y la observación personal tienen el nivel de evidencia más bajo ${ }^{12-14}$.

\section{Estudios observacionales analíticos}

Constituyen un nivel superior de evidencia científica. Se distinguen: los estudios de casos y controles, y los estudios de cohortes.

Los estudios de casos y controles suelen ser diseños clínicos de carácter retrospectivo y se enfocan sobre todo al análisis causal y a la relación de factores de riesgo en el desarrollo de una enfermedad. Los pacientes entran en el estudio en base a la variable resultado o dependiente (tener o no tener la enfermedad o condición patológica) y retrospectivamente se analiza el factor bajo estudio o variable independiente (VI). Por ejemplo, en un estudio de casos-control sobre la relación entre trastornos del sueño y síndrome del túnel carpiano (STC), se divide la frecuencia de la VI (trastorno del sueño) en los que presentaban STC entre la frecuencia de disturbios del sueño en los no afectos de STC obteniendo los resultados en base al «odds ratio» ${ }^{12-14,19}$.

En los estudios de cohortes, los pacientes entran en el diseño clínico en base a la VI o de exposición. Así tendremos una población de disturbios del sueño y otra no afecta de disturbios del sueño; se siguen longitudinalmente en el tiempo, y se analiza la incidencia de STC en los expuestos partida por la incidencia de STC en los no expuestos, mostrando los resultados en base al «riesgo relativo». El seguimiento longitudinal se puede hacer de manera prospectiva (estudio de cohortes clásico) o de forma retrospectiva (estudio de cohortes histórico). Característicamente, siempre un diseño de cohortes tiene más evidencia que un diseño de casos-control en estudios sobre causalidad o etiológicos.

Los diseños de cohortes también se emplean en trabajos de valoración de resultados outcomes, pudiendo alcanzar el nivel 2 de evidencia científica si se tiene un seguimiento del más del $80 \%$ de la población, siendo el diseño de 


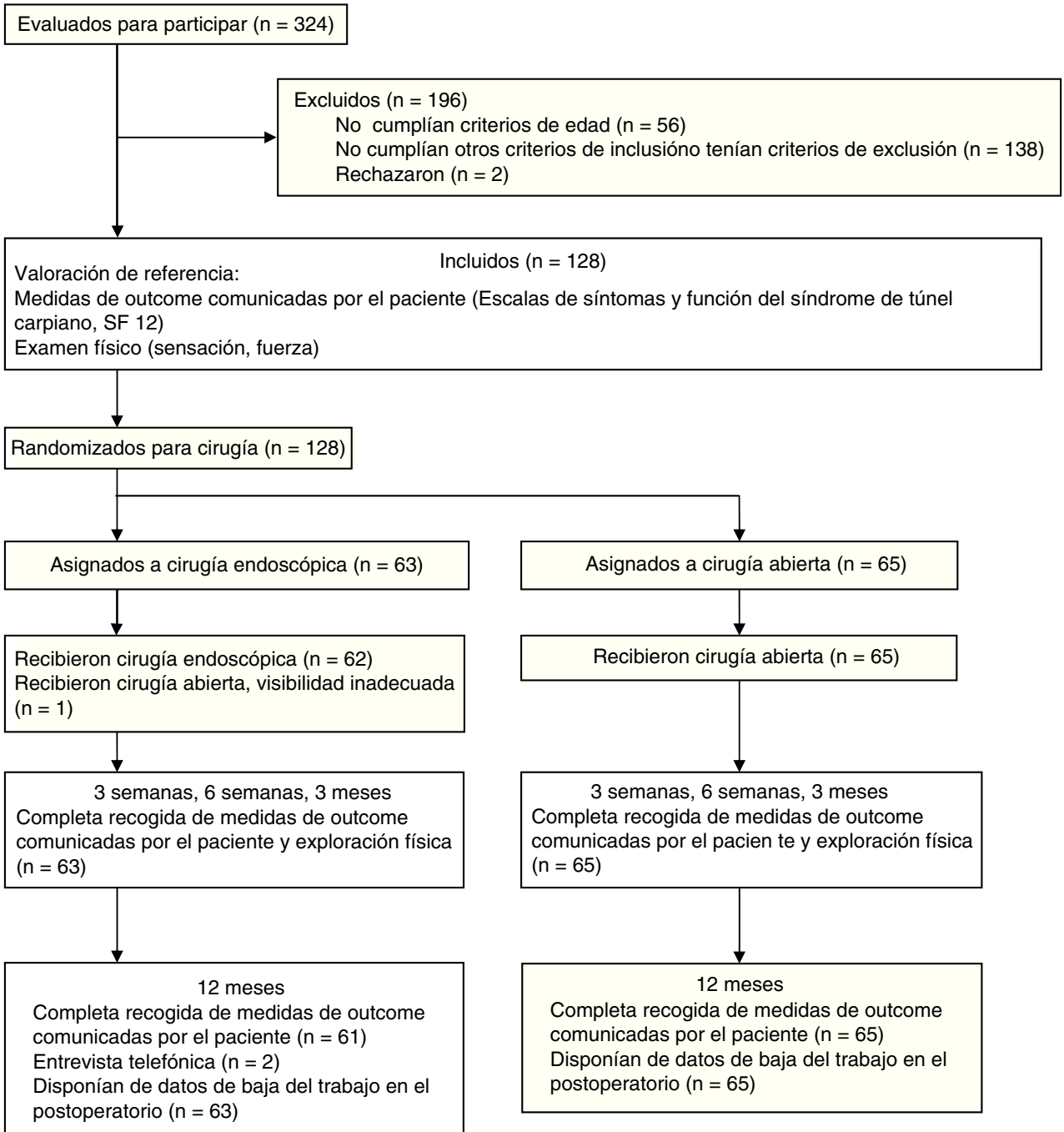

Figura 2 Diagrama de flujo en un ensayo clínico aleatorizado. Fuente: adaptado de Atroshi et al. ${ }^{35}$.

elección cuando queremos analizar la efectividad de un solo tratamiento o técnica quirúrgica (fig. 1). Estos estudios se denominan también como «estudios antes-después», y se caracterizan por tener al menos 2 mediciones en el tiempo, una antes del tratamiento y otra después, calculándose los resultados en base al tamaño del efecto (ES) y a la media estandarizada de la respuesta (SRM).

Finalmente, los diseños de cohortes también se pueden emplear en los estudios pronósticos, donde los autores intentan analizar qué características de los pacientes influyen en el resultado final u outcomes. Estos estudios suponen el mayor nivel de evidencia o nivel 1. Un ejemplo claro del uso de los diseños de cohortes en estudios pronósticos lo constituye el trabajo de Bolgrenn et al. ${ }^{20}$ donde se analiza el cómo la mala unión de fracturas distales de radio se asocia a discapacidades en miembros superiores (MMSS) tardías, hasta 2 años después del traumatismo.

Estudios experimentales o ensayos clínicos aleatorizados Cuando queremos comparar 2 tratamientos, el ECA es el diseño clínico con mayor evidencia científica, solo superado por las RS. Según la escala de Jaddad modificada, que se utiliza para valorar la calidad de los ECA en cirugía de los MMSS $^{21}$, con una puntuación de «0» (peor) a «5» (mejor), 3 son los puntos importantes a tener en cuenta a la hora de definir un ECA: la aleatorización (tipo, quién la realizó, método de aleatorización y asignación de los pacientes), el enmascaramiento, y las pérdidas prealeatorización y postaleatorización (fig. 2) 2 $^{22}$. Para mejorar la calidad en la presentación y publicación de los ECA, los editores de revistas científicas establecieron las normas del CONSORT que conforman un listado a revisar antes de enviar un ECA a una revista científica médica. La RICMA recomienda a los autores seguir las indicaciones del CONSORT en sus normas editoriales.

Metaanálisis y revisiones sistemáticas

El mayor nivel de evidencia posible la tienen estos diseños clínicos. Su publicación en revistas internacionales de Cirugía de la Mano es muy infrecuente $\mathrm{e}^{12,17}$. Las RS y MA permiten la síntesis de la evidencia científica ya publicada en ECA y en menor medida en estudios observacionales. A la hora de presentar un MA o una RS es necesario seguir las estrategias de 
Prisma 2009. Diagrama de flujo.

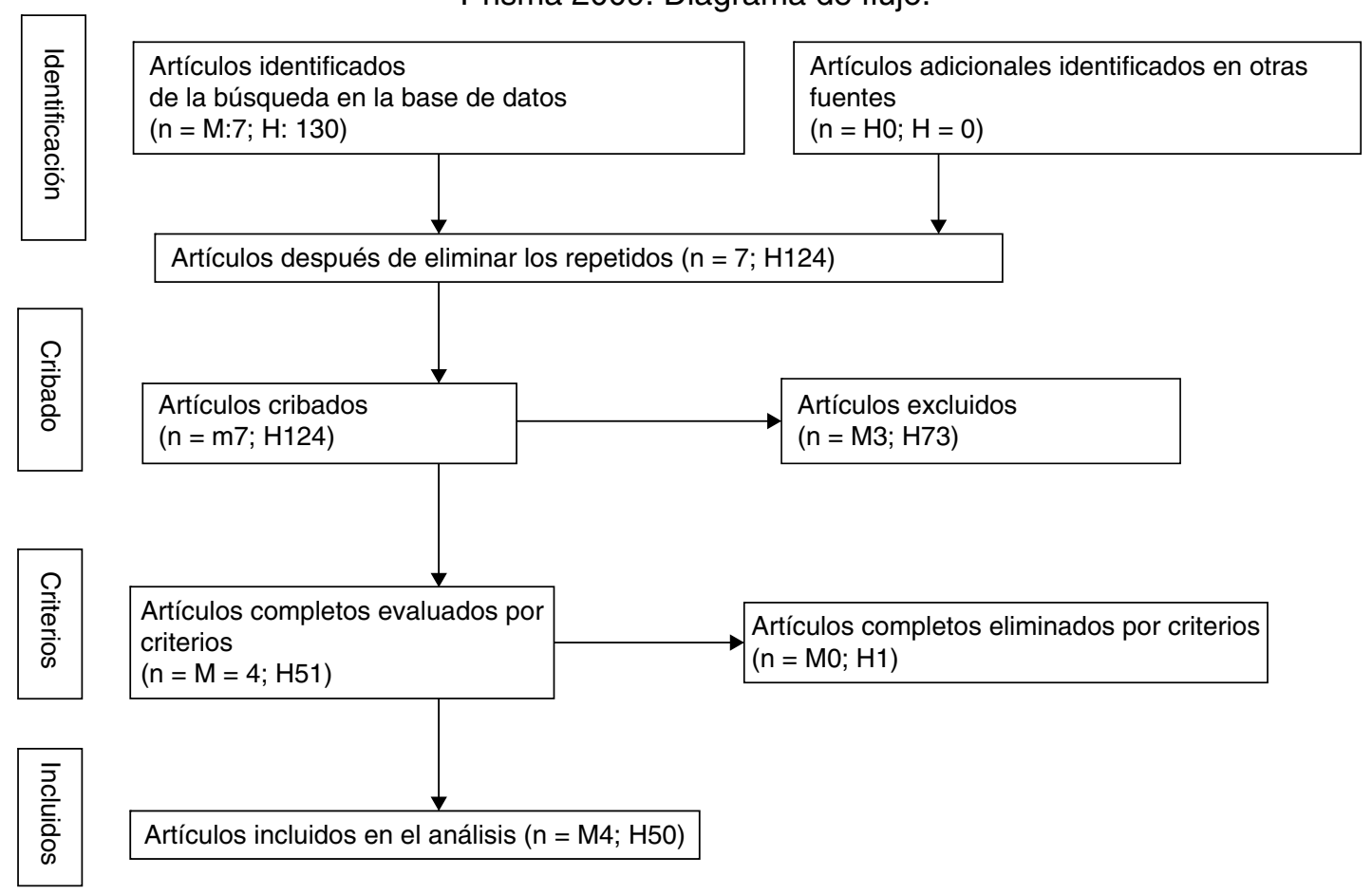

Figura 3 Diagrama de flujo en una revisión sistemática. Ensayo clínico aleatorizado en artroscopia de muñeca comparado con artroscopia de hombro; mostrado en un diagrama de flujo de PRISMA.

H: número de artículos de artroscopia de hombro; M: número de artículos de artroscopia de muñeca.

Fuente: adaptado de Tadjerbashi et al. ${ }^{24}$.

la revisión propuestas en la guía Preferred Reporting Items for Systematic Reviews and Meta-Analyses (PRISMA) ${ }^{23}$, aportando diagramas de flujo, variables outcomes y método de la valoración o síntesis de la evidencia (fig. 3) ${ }^{24}$.

Generalmente, una RS es superior a cualquier estudio individual. Dentro de los estudios individuales, un estudio experimental (e.g.: ECA de buena calidad) es normalmente mejor que cualquier estudio observacional. Dentro de los estudios observacionales, un estudio de cohorte es mejor que cualquier estudio de casos-control. Un estudio de casos-control es generalmente mejor que cualquier estudio de serie de $\operatorname{casos}^{17,18}$.

En ocasiones, un trabajo de investigación clínica puede tener más de un diseño clínico debido a la existencia de más de una pregunta de investigación. En ese caso hay que expresar de menor a mayor evidencia los diseños clínicos empleados, siguiendo las preguntas o propósitos establecidos en la sección «introducción». Por ejemplo, en un estudio sobre la versión española del Patient Rated Wrist Evaluation (PRWE) $)^{15}$ se establecieron como propósitos el analizar la fiabilidad, la validez y la respuesta del PRWE a la hora de valorar resultados en fracturas distales de radio. En ese caso, la correcta descripción del diseño clínico sería: «Para el análisis de la validez de construcción se utilizó un diseño clínico de corte transverso. Se realizó el estudio en dos momentos distintos del seguimiento. El primero, a nivel basal (baseline), cuando se esperaba el mejor estado de salud y de discapacidad en los pacientes. El segundo, a las ocho semanas, cuando se esperaba la mayor discapacidad y el peor estado de salud tras la fractura. En el análisis de la fiabilidad y la respuesta, se empleó un diseño clínico longitudinal prospectivo o cohortes clásico con mediciones hechas a nivel basal, a las ocho, nueve, doce y trece semanas» (fig. 1) ${ }^{15}$.

\section{Instrumentos y medidas}

La Clasificación Internacional de la Función, Discapacidad y Salud (ICF) fue desarrollada por la Organización Mundial de la Salud para ser aplicada en distintos aspectos de la salud (http://www3.who.int/icf). Las medidas de los resultados pueden clasificarse de acuerdo con la clasificación ICF de la OMS en función corporal y estructura, actividad y participación. Tradicionalmente las medidas utilizadas para evaluar los resultados en el campo de la Cirugía de la Mano han sido principalmente medidas de la función corporal y estructura, como por ejemplo, radiografías, medida de fuerza o amplitud de movimiento. Recientemente los instrumentos de salud, como el cuestionario DASH, Michigan Hand Questionnaire, PRWE, etc. han sido introducidos en la Cirugía de la Mano para estudios de evaluación de efectos del tratamiento ${ }^{12,21}$ como medidas de la participación y la actividad.

Los autores deben definir en esta subsección del «método», las variables resultados outcomes primarias y cuáles van a ser las secundarias. Para ello, se debe justificar con citas bibliográficas el empleo de las mismas, lo que vendrá dado por el nivel de respuesta o sensibilidad para detectar cambios de importancia clínica. 
En caso de emplear medidas de la estructura y el cuerpo (fuerza, rango de movimiento, dos puntos de discriminación sensitiva, etc.) como variables resultados, se debe explicar: quién realizó las mediciones y si se realizaron análisis de la fiabilidad (error intraobservador y error interobservadores) y de la validez de dichas mediciones. En caso negativo, aportar citas bibliográficas sobre la fiabilidad y validez de dichas medidas en la literatura científica. Destacar la importancia de describir claramente las entidades que estamos analizando en la subsección de «instrumentos y medidas»: pacientes versus manos versus dedos. Por ejemplo, supongamos que los autores quieren comparar los resultados de 2 prótesis de metacarpofalángicas (MCF) en pacientes reumáticos mediante un ECA. Sabiendo que la enfermedad no afecta todas las articulaciones MCF por igual, y que existe variabilidad de una mano a otra en pacientes afectos bilateralmente, ¿qué unidad de análisis fue empleada a la hora de usar medidas de la función y estructura como fuerza o rango de movilidad activa?, ¿pacientes, manos o dedos? ${ }^{25}$. Este apartado es fundamental para dejar claro a los lectores del trabajo de investigación qué unidades son las que van a ser comparadas, y si se cumple el principio de independencia de las mediciones.

El anterior problema (variabilidad entre pacientes, manos y dedos en relación con la independencia de las mediciones) no afecta al estudio cuando usamos medidas estándares de la participación y actividad mediante los instrumentos Patients Reported Outcomes (PRO). En este caso se recomienda en esta subsección del «método» describir adecuadamente los instrumentos PRO usados, justificar su uso, qué dimensión de la salud miden, cómo se puntúan, cómo se manejan las pérdidas en las contestaciones de los ítems, el rango de las puntuaciones (e.g.: para el DASH, que mide discapacidad en MMSS, da las puntuaciones en un rango de «0» menor discapacidad, a «100» mayor discapacidad. El EQ-5D Index, que mide calidad de vida, da sus puntuaciones en un rango de «0» la muerte, a « 1 » mejor calidad de vida y salud), y finalmente, aportar la cita bibliográfica de la versión oficial adaptada al español (España) o bien acceso a la web oficial de la institución que desarrolló el instrumento donde se avala dicha versión oficial y estándar en español. Al igual que en las medidas de la función corporal y estructura, cuando se usan instrumentos PRO, hay que aportar citas bibliográficas sobre la fiabilidad, validez y respuesta para su uso en la población diana de nuestro trabajo y para contestar una pregunta concreta de investigación.

\section{Análisis estadístico}

\section{Clasificación y descripción de las variables}

En este punto debemos comenzar definiendo todas las variables de las que hemos recogido datos, y clasificarlas de una forma matemática. Así, las variables numéricas o cuantitativas son aquellas que pueden cuantificarse. Se pueden dividir en: a) discretas, si no admiten tomar todos los valores intermedios en un rango, tomando con frecuencia valores enteros (número de hijos o número de partos), y b) continuas, si admiten tomar cualquier valor dentro de un rango determinado (edad, peso, índice de masa corporal, puntuación del DASH, fuerza, rango de movimiento, etc. ). Las variables cualitativas (o categóricas) representan una cualidad o atributo que clasifica a cada individuo en una o varias categorías. Se dividen en: a) ordinales, si las categorías son susceptibles de ser ordenadas de modo lógico (clase social: baja, media, alta), y b) nominales, si las categorías no siguen ningún orden natural (grupo sanguíneo: $A, B, A B, 0$ ). Las variables cualitativas que solo aceptan 2 posibilidades se denominan dicotómicas (género: hombre/mujer). Del autor depende la correcta descripción del cómo se han codificado las distintas variables estudiadas. Así, una variable continua como la edad se puede convertir en una variable categórica separando en intervalos la variable edad (por ej.: < 30 años, $30-45,46-60,>60$ años) ${ }^{13}$.

\section{Exploración de los datos}

Los autores deben justificar de manera clara el uso de test paramétricos o no paramétricos en su análisis en base a la exploración de la normalidad y la homocedasticidad de las varianzas de sus datos. Por ejemplo: «Las puntuaciones de los tres instrumentos PRO, PRWE versión española bajo estudio, QuickDASH (Rosales et al., 2002 y 2009) y EuroQol-5D (EuroQol Group, 1990), fueron exploradas para conocer si seguían una distribución normal mediante el test de Kolmogorov-Smirnov y de Shapiro-Wilk en las diferentes administraciones del diseño clínico de cohortes (nivel basal, ocho, nueve, doce y trece semanas). Se estableció la hipótesis nula $(\mathrm{H} 0)$ de que no existía diferencia significativa entre la muestra y la población normal, utilizando un nivel de significación (alfa) de 0,05. Las diferentes muestras fueron también analizadas para la homocedasticidad de las varianzas mediante el test de Levene con un nivel de significación (alfa) de 0,05. Se estableció la hipótesis nula (H0) de que las varianzas eran homogéneas» ${ }^{15}$.

De manera similar, el uso de los análisis ANOVA cuando tenemos más de 2 muestras de datos a comparar hay que justificarlo. ANOVA es un estadístico muy usado tanto en la investigación experimental como en los estudios biomecánicos, y de manera generalizada y en muy pocos trabajos se justifica su uso. Es un estadístico altamente demandante y se debe demostrar la normalidad, homocedasticidad e independencia de los errores como paso previo a su uso.

\section{Descripción de los test de análisis empleados}

Los autores deben describir los test de análisis empleados, definiendo el nivel de significación (alfa) y estableciendo la hipótesis nula de su análisis estadístico (e.g.: «Las muestras fueron analizadas con la prueba estadística t-Student para muestras apareadas en los casos en los que la variable seguía una distribución normal y una homogeneidad de las varianzas. Se utilizó un nivel de significación del 0,05, estableciéndose la hipótesis nula $(\mathrm{HO})$ de que la media de la diferencia en las puntaciones de los instrumentos PRO era igual a cero en los intervalos nivel basal-8 semanas, 8-9 semanas, $9-12$ semanas y $12-13$ semanas $»^{15}$ ).

Un error importante ocurre cuando tras ser usados test no paramétricos, debemos describir los test estadísticos empleados, como el test de Wilconxon para comparar 2 muestras relacionadas o el test de U Mann-Whitney para comparar 2 muestras no relacionadas. En esos casos es frecuente que los autores presenten sus hipótesis nulas hablando de media y desviación estándar cuando los test no paramétricos comparan medianas y rangos intercuartiles. 
Una manera correcta de describir el uso de test no paramétricos podría ser: «Se utilizó el test de Wilconxon para muestras relacionadas estableciéndose la hipótesis nula $(\mathrm{H} 0)$ de que la mediana de las diferencias en las puntuaciones era igual a cero. Este test no paramétrico se utilizó en todos los intervalos del instrumento EuroQol-5D y el intervalo nivel basal-8 semanas para el PRWE y QuickDash» ${ }^{15}$.

\section{Tamaño muestral y poder del análisis}

Los autores deben describir si se hizo una valoración del poder del análisis y cálculo a priori del tamaño muestral. Esta área suele ser muy deficitaria tanto en los estudios de investigación clínica como experimentales ${ }^{7}$. En algunos casos, si los revisores del trabajo le pidieran a los autores información sobre este apartado, siempre se puede incluir un comentario en la discusión como limitación del trabajo como por ejemplo «se realizó un análisis post hoc del poder del estudio».

\section{Cómo escribir los resultados}

En la sección «resultados» de un artículo, los autores deben exponer sencillamente «lo descubierto», «los hechos». El error más común, incluso observado en manuscritos enviados a revistas importantes, es la inclusión de comentarios que intentar aclarar o explicar los resultados. Ese tipo de comentarios pertenecen a la sección «discusión». Los «resultados» no es el lugar del artículo donde debamos incluir citas bibliográficas o bien la interpretación de los datos. Los autores deben establecer los puntos fundamentales de los resultados con datos pero no con argumentos ${ }^{26}$.

Como se comentó anteriormente, algunas revistas o editores prefieren que la sección de «resultados» comience con una descripción de la muestra estudiada y sus propiedades demográficas. Nosotros creemos que esta información pertenece a la sección «método» cuando definimos nuestra población estudio. Se deben describir los resultados siguiendo el orden de análisis expuesto en el método, pudiéndose complementar con tablas o figuras. No podemos repetir o duplicar información incluida ya en tablas, que tienen que ser lo suficientemente claras para ser entendidas sin necesidad de acudir al texto del manuscrito.

La sección «resultados» se debe escribir en tiempo pasado. No usar palabras como «significante» a no ser que sea para describir una significación o diferencia estadísticamente significativa. No use esa palabra como sinónimo de «importante». En caso de resultados no significativos, no intente justificarlos usando frases como «los datos muestran una tendencia a la mejoría...». Normalmente, la palabra «tendencia» implica un estudio con un pobre diseño clínico, excepto en los diseños clínicos ecológicos 9 .

\section{Cómo escribir la discusión}

La sección «discusión» del trabajo científico es el lugar donde los autores interpretan sus resultados. Toda discusión debe incluir los siguientes subapartados: 1) resumen de los hallazgos más importantes, 2) comparación e interpretación, 3) relevancia clínica, 4) puntos fuertes y débiles (limitaciones del estudio) de su trabajo, y 5) propuesta de nuevos estudios si fuera necesario.

\section{Hallazgos principales}

La discusión debe comenzar con un simple párrafo que resuma los hallazgos principales o los más importantes de su estudio. Contestar si se rechazó la hipótesis nula con sus hallazgos. En caso contrario, no ser catastrofistas (e.g.: no encontrar diferencias estadísticamente significativas no implica que los autores no puedan publicar su trabajo), aunque haya sido descrito que trabajos con resultados negativos son mucho más difíciles de ser aceptados en comparación a los trabajos con resultados positivos, que suelen ser aceptados en un tercio de los casos en la mayoría de los journals. Es importante, en el caso de obtener resultados negativos, el haber analizado el tamaño muestral y el error tipo ॥ (obtener un resultado no significativo cuando realmente si lo hay debido a un tamaño muestral muy pequeño). De hecho, Chung et al. ${ }^{27}$ demostraron que el $82 \%$ de los estudios negativos publicados en los journals más importantes de la mano tenían un poder estadístico inadecuado para sustentar sus conclusiones.

\section{Interpretación y comparación}

Una vez descritos los resultados más significativos, el siguiente párrafo de la discusión debe ser dedicado a comparar dichos resultados con los datos que estén previamente publicados. Para ello se puede utilizar el tiempo presente cuando se escriba sobre conocimientos establecidos por otros autores, mientras que para escribir sobre su trabajo o resultados, los autores deben usar el tiempo pasado.

\section{Relevancia clínica}

En el tercer párrafo de la discusión, los autores deben establecer la relevancia clínica de sus hallazgos y su relación con la pregunta de investigación e hipótesis establecidas en la introducción. No usar frases como «Este es el primer estudio...»0 «Se ha demostrado por primera vez...». Raramente en la ciencia un trabajo cambia el curso del conocimiento científico con un solo artículo. Los autores no deben mostrarse en la escritura sobre-entusiasmados con sus resultados.

\section{Limitaciones y puntos fuertes del trabajo}

El párrafo final de la discusión debe dejar claro cuáles son los puntos fuertes y débiles del trabajo. Kotsis y Chung ${ }^{9}$ prefieren escribir primero sobre las limitaciones y a continuación finalizar con los puntos fuertes del trabajo, ya que de esta manera dejamos al lector una buena nota o «buen sabor de boca» al final, cuando esté terminando de leer el trabajo. Sin embargo, nosotros recomendamos escribir primero sobre los puntos fuertes del trabajo y terminar con las limitaciones, pues ello permite a los revisores dejar claro la honestidad de los autores y, de camino, enlazar la fase final de la discusión con la proposición de nuevos estudios que puedan aclarar en el futuro los puntos débiles reconocidos por los autores en el trabajo. 


\section{Escribir el abstract}

Ahora es el momento de escribir el abstract, la parte más leída junto con el título de su trabajo de investigación. Es la parte que le llega a los revisores imparciales y que leen antes de aceptar o rechazar la revisión del artículo. Un abstract mal escrito implica un mal artículo o una falta de habilidad o formación para la escritura científica, aunque haya sido un trabajo científico sensacional y durísimo en el tiempo. Solicitad ayuda a un colega más experto que corrija vuestro abstract antes de enviarlo a la revista. Nosotros en nuestro grupo solemos hacerlo y, por ejemplo, en caso de tener problemas a la hora de reducir el abstract al máximo de palabras requeridas por la editorial, pedimos a uno de nuestros compañeros que lo revise. Es la parte del artículo que más tiempo debemos emplear en su elaboración. Revisar una y otra vez las normas de autor de la revista, que en ocasiones cambian durante el año en curso, y establecer claramente la longitud en palabras del abstract que requiera la revista. Algunos journals como el JHSE prefieren que no se incluyan datos estadísticos. Personalmente nosotros creemos que el abstract debe ser lo más explicativo posible con los datos más importante desde el punto de vista científico, siendo obligado el aportar información estadística. De hecho, las revistas de gran impacto como The Lancet, JAMA, BMJ, New England Journal of Medicine, Nature, Science, prefieren un abstract con los datos más explícitos posibles, utilizando números y estadísticas ${ }^{28}$.

Escribir un abstract implica extraer y resumir (ABabsolutely, STR-straightforward, ACT-actual data). Las primeras 2 frases son destinadas al «qué no se sabe sobre el tema (pregunta de investigación)» y el propósito del trabajo. Intentar resumir y comprimir el mensaje (e.g.: «el propósito del presente trabajo fue valorar la efectividad de la liberación endoscópica del ligamento transverso del carpo en el tratamiento del STC»).

A continuación «el método». Se debe escribir una oración o frase para cada una de las partes del método (población estudio, diseño clínico, variables outcomes y análisis). Por ejemplo: «En este estudio de cohortes clásico, un total de 90 pacientes con una edad media de 54 años (63 mujeres) con el diagnostico de STC en base a criterios clínicos y neurofisiológico completaron los instrumentos CTS-6 y QuickDASH el día antes y 3 meses después de la cirugía. La efectividad se analizó en base al tamaño del efecto (ES) y la media estandarizada de la respuesta (SRM)».

Los «resultados» es la parte más importante del abstract. Se deben presentar los resultados de forma clara, evitando frases largas y confusas, y siguiendo la simple lógica. La presentación precisa de los datos en el abstract es extremadamente importante. Una revisión reciente de 243 abstract publicados en «journals selectos de farmacoterapia» demostró que un $25 \%$ presentaban omisión de datos y un $60 \%$ de los abstracts fueron clasificados como deficientes a la hora de presentar sus resultados de una forma precisa y exacta ${ }^{29}$. En el ejemplo anterior sobre le efectividad de la liberación endoscópica del STC, mediante un diseño de cohortes clásico con medidas antes de la cirugía y 3 meses después, es necesario un paso previo en el análisis de la efectividad que sería el analizar si las diferencias en las puntuaciones de antes a después de la cirugía de las variables outcomes (CTS-6 y QuickDASH) fueron significativas, ya sea mediante un t-Student - test para muestras apareadas - o mediante un test de Wilconxon - test para muestras relacionadas en base a la normalidad y homocedastcidad de las varianzas de los datos-. Incluir toda esta información en el abstract es innecesario. Lo que hay que incluir son aquellos resultados que nos den información sobre qué diferencias estadísticas tenían importancia clínica, y por ello se incluyen solo en el abstract los resultados del análisis del efecto del tratamiento (e.g.: «Se objetivó una importante mejoría clínica a nivel de los instrumentos CTS-6 [ES = 1,2, SRM = 1,4] y QuickDASH [ES = 0,9, SRM = 1,1]»).

Finalmente, el abstract debe terminar con una frase que resuma su conclusión más importante en relación con la pregunta de investigación, evitando hacer comentarios o interpretaciones (e.g.: «La liberación endoscópica es un procedimiento efectivo en mejorar los síntomas y la discapacidad de MMSS en pacientes afectos de STC»).

Así, nuestro abstract de un estudio de cohortes clásico para medir la efectividad de la liberación endoscópica en el tratamiento del STC quedaría de la siguiente manera, con total de 135 palabras:

Objetivo: el propósito del presente trabajo fue valorar la efectividad de la liberación endoscópica del ligamento transverso del carpo en el tratamiento del STC.

Método: en este estudio de cohortes clásico, un total de 90 pacientes con una edad media de 54 años (63 mujeres) con el diagnostico de STC en base a criterios clínicos y neurofisiológico completaron los instrumentos CTS-6 y QuickDASH el día antes y 3 meses después de la cirugía. La efectividad se analizó en base al tamaño del efecto (ES) y la media estandarizada de la respuesta (SRM).

Resultados: se objetivó una importante mejoría clínica a nivel de los instrumentos CTS-6 $(E S=1,2, S R M=1,4)$ y QuickDASH $(\mathrm{ES}=0,9, \mathrm{SRM}=1,1)$.

Conclusión: la liberación endoscópica es un procedimiento efectivo en mejorar los síntomas y la discapacidad de MMSS en pacientes afectos de STC.

\section{Escribir el título}

Un buen título debería ser escrito de forma clara, corta y específica. El título debe reflejar el propósito, estableciendo el tópico que se abordó con la investigación, en vez de reflejar las conclusiones del estudio. No se deben usar títulos en forma de preguntas ${ }^{10}$ como en esta traducción de un artículo publicado en el JHSE «¿Qué cuestionario es mejor? Fiabilidad, validez y facilidad de uso del PEM, DASH y MHQ ${ }^{30}$. Es más correcto evitar preguntas, frases que contengan palabras llamativas, pegajosas y demasiados elocuentes ${ }^{10}$, como en el siguiente trabajo publicado también en el JHSE sobre el mismo tópico (instrumentos de medición) por nuestro equipo de trabajo: «Respuesta relativa y validez de construcción de la versión española del DASH para valorar resultados en STC» ${ }^{31}$.

Fundamental en el título es hacer mención a 3 puntos: propósito, población y diseño clínico. Si es posible, lo más corto posible. De hecho, una revisión reciente del 2015 ha demostrado que, aparte del impacto de la revista de 
Tabla 1 Ejemplo a incluir en la escritura científica de un artículo original en Cirugía de la Mano

\begin{tabular}{|c|c|c|}
\hline Sección & Elemento & Ejemplo \\
\hline Introducción & Propósito & $\begin{array}{l}\text { El propósito de este artículo fue comparar la liberación abierta o endoscópica del túnel } \\
\text { carpiano en los pacientes trabajadores con síndrome del túnel carpiano en relación con } \\
\text { el dolor postoperatorio, calidad de vida y duración de la baja laboral después de la } \\
\text { intervención }\end{array}$ \\
\hline \multirow{8}{*}{$\begin{array}{l}\text { Material } \\
\text { y métodos }\end{array}$} & \multicolumn{2}{|c|}{ A. Definición de la población a estudio } \\
\hline & $\begin{array}{l}\text { Criterios de } \\
\text { elección y control } \\
\text { de las variables de } \\
\text { confusión }\end{array}$ & $\begin{array}{l}\text { Criterios de inclusión: síndrome del túnel carpiano (STC) primario idiopático, edad } \\
\text { de } 25-60 \text { años, actualmente empleado, duración de los síntomas de al menos } 3 \text { meses, } \\
\text { respuesta inadecuada al tratamiento con ortesis de muñeca durante } 3 \text { semanas, } \\
\text { síntomas de probable o clásico STC de acuerdo con los criterios diagnósticos del } \\
\text { diagrama de la mano de Katz, y estudios de conducción nerviosa que mostraban } \\
\text { neuropatía del nervio mediano en la muñeca (latencia motora distal } \geq 4,5 \text { ms, latencia } \\
\text { sensitiva muñeca-dedo } \geq 3,5 \mathrm{ms,} \mathrm{o} \mathrm{velocidad} \mathrm{de} \mathrm{conducción} \mathrm{sensitiva} \mathrm{en} \mathrm{el} \mathrm{segmento} \\
\text { del túnel carpiano < } 40 \mathrm{~m} / \mathrm{s} \text { ). Criterios de exclusión: enfermedad inflamatoria articular, } \\
\text { diabetes mellitus, enfermedad tiroidea, gestación, trauma sobre la mano afecta } \\
\text { durante el año precedente, cirugía previa de liberación del túnel carpiano en la mano } \\
\text { afecta, cirugía de liberación del túnel carpiano en la mano contralateral durante el año } \\
\text { precedente, síntomas de STC en la mano contralateral no aliviados adecuadamente } \\
\text { mediante ortesis en el momento del reclutamiento, baja laboral actual por dolencia } \\
\text { distinta a síndrome de túnel carpiano, e incapacidad para completar cuestionarios } \\
\text { debido a problemas lingüísticos o cognitivos }\end{array}$ \\
\hline & $\begin{array}{l}\text { Reclutamiento de } \\
\text { los pacientes }\end{array}$ & $\begin{array}{l}\text { El estudio se realizó en el departamento de Cirugía Ortopédica de un solo centro } \\
\text { hospitalario con un área de referencia de } 170.000 \text { habitantes. Los pacientes fueron } \\
\text { reclutados de entre aquellos que eran remitidos por los médicos de atención primaria } \\
\text { con síntomas de STC. Los pacientes elegibles fueron enrolados por el cirujano } \\
\text { ortopédico que los examinaba. Cada paciente recibió una información verbal y escrita } \\
\text { completa acerca del ensayo, obteniendo el consentimiento informado. Solo se permitió } \\
\text { a los pacientes entrar en el estudio una vez }\end{array}$ \\
\hline & $\begin{array}{l}\text { Aleatorización y } \\
\text { asignación de } \\
\text { tratamientos }\end{array}$ & $\begin{array}{l}\text { Los pacientes reclutados fueron programados para la cirugía por una enfermera y } \\
\text { fueron asignados a un grupo de tratamiento en el quirófano inmediatamente antes de la } \\
\text { cirugía, de acuerdo con la aleatorización generada por un ordenador en bloques de } 8\end{array}$ \\
\hline & Enmascaramiento & $\begin{array}{l}\text { En el quirófano, el cirujano (que no tenía conocimiento del tamaño de los bloques de } \\
\text { aleatorización), abría el sobre sellado opaco con la numeración más baja que contenía } \\
\text { el tipo de intervención quirúrgica a realizar }\end{array}$ \\
\hline & $\begin{array}{l}\text { Pérdidas pre y } \\
\text { postaleatorización }\end{array}$ & $\begin{array}{l}\text { No existieron pérdidas o abandonos en el estudio. Dos pacientes (grupo cirugía } \\
\text { endoscópica) no devolvieron los cuestionarios a los } 12 \text { meses del CTS y SF-12. Fueron } \\
\text { entrevistados de forma telefónica. Cuando al menos un ítem se dejó sin contestar, el } \\
\text { cuestionario SF-12 no pudo ser calculado en } 4 \text { pacientes de forma preoperatoria (uno } \\
\text { del grupo cirugía abierta y } 3 \text { del grupo cirugía endoscópica), y en } 2 \text { pacientes a los } \\
12 \text { meses (uno de cada grupo) }\end{array}$ \\
\hline & B. Diseño clínico & Ensayo clínico aleatorizado en un único departamento de Cirugía Ortopédica \\
\hline & $\begin{array}{l}\text { C. Instrumentos } \\
\text { y medidas }\end{array}$ & $\begin{array}{l}\text { Los pacientes fueron evaluados con instrumentos específicos de valoración de } \\
\text { resultados, cuestionarios de calidad de vida y exploración física a las } 3 \text { y } 6 \text { semanas, } \\
\text { y a los } 3 \text { y } 12 \text { meses después de la cirugía. La variable de resultados primaria fue la } \\
\text { severidad del dolor postoperatorio en la cicatriz y la región palmar proximal y el grado } \\
\text { en que esta limitaba las actividades. Para su medición se tomó una escala de dolor de } \\
2 \text { ítems (adaptada de la escala de dolor corporal del cuestionario SF-36). Como } \\
\text { variables de resultados secundarias se utilizaron el cuestionario de síndrome de túnel } \\
\text { carpiano o CTS, el SF-12, el tiempo de baja laboral y la exploración física }\end{array}$ \\
\hline Resultados & & $\begin{array}{l}\text { El dolor en la cicatriz o a nivel palmar proximal fue menos prevalente o severo después } \\
\text { de la cirugía endoscópica que en la cirugía abierta, pero las diferencias fueron } \\
\text { pequeñas. A los } 3 \text { meses el dolor en la cicatriz o en la palma de la mano fue informado } \\
\text { en el } 52 \% \text { de pacientes del grupo endoscópico y en el } 82 \% \text { del grupo de cirugía abierta, } \\
\text { y la diferencia de puntuación media para la severidad del dolor en la cicatriz o en la } \\
\text { palma de la mano y la limitación en las actividades fue de } 13,3 \text { (5,3-21,3). No se } \\
\text { encontraron diferencias entre los grupos en el resto de resultados. La media de baja } \\
\text { laboral después de la cirugía fue de } 28 \text { días en ambos grupos. Las medidas de la calidad } \\
\text { de vida mejoraron de manera substancial }\end{array}$ \\
\hline
\end{tabular}


Tabla 1 (continuación)

\begin{tabular}{|c|c|c|}
\hline Sección & Elemento & Ejemplo \\
\hline \multirow[t]{5}{*}{ Discusión } & $\begin{array}{l}\text { Hallazgos } \\
\text { principales }\end{array}$ & $\begin{array}{l}\text { La cirugía endoscópica en el síndrome del túnel carpiano produce menos dolor } \\
\text { postoperatorio en la cicatriz y región palmar proximal y menor limitación de la } \\
\text { actividad que la cirugía abierta, pero con pequeñas diferencias }\end{array}$ \\
\hline & Interpretación & $\begin{array}{l}\text { Este estudio provee una fuerte evidencia de que la liberación endoscópica del túnel } \\
\text { carpiano produce un alivio de los síntomas y una mejora en la calidad de vida } \\
\text { relacionada con la salud similar que la liberación abierta }\end{array}$ \\
\hline & Relevancia clínica & $\begin{array}{l}\text { La cirugía endoscópica se asocia con menor dolor que la cirugía abierta de forma } \\
\text { modesta hasta los } 3 \text { meses postoperatorios, pero no muestra ventajas en lo que } \\
\text { respecta al tiempo de baja laboral. Ambos métodos tienen la misma eficacia en el alivio } \\
\text { de los síntomas del síndrome del túnel carpiano. El pequeño tamaño del beneficio } \\
\text { clínico hace que el coste efectividad de la cirugía endoscópica sea incierto }\end{array}$ \\
\hline & Limitaciones & $\begin{array}{l}\text { Los } 2 \text { métodos no difirieron en la tasa de complicaciones, pero fue necesario } \\
\text { reintervenir } 2 \text { pacientes después de la cirugía endoscópica y un paciente tras la cirugía } \\
\text { abierta. Sin embargo, ha crecido la preocupación acerca del riesgo de complicaciones } \\
\text { en la cirugía endoscópica donde la incidencia manifestada de complicaciones graves, } \\
\text { como lesión nerviosa irreversible, ha sido baja (ninguno en los estudios aleatorizados y } \\
\text { menos del } 2 \% \text { en los estudios observacionales). Por lo tanto se necesitaría una muestra } \\
\text { más grande en un ensayo para detectar las posibles diferencias en la tasa de } \\
\text { complicaciones }\end{array}$ \\
\hline & Fortaleza & $\begin{array}{l}\text { Hasta nuestro conocimiento, este es el estudio aleatorizado más grande que compara } \\
\text { dolor y retorno al trabajo después de la cirugía abierta o endoscópica del síndrome del } \\
\text { túnel carpiano. Los hallazgos de nuestro ensayo aleatorizado deberían ser aplicados a } \\
\text { otros países puesto que el ensayo comprende una comparación aleatorizada de } 2 \text { grupos }\end{array}$ \\
\hline
\end{tabular}

Fuente: adaptada de Atroshi et al. ${ }^{35}$.

publicación, uno de los factores más importantes que se asocia a un mayor número de citaciones de dicho trabajo es un título más corto ${ }^{32}$.

\section{El futuro de la investigación clínica en Cirugía de la Mano}

Los mejores trabajos en Cirugía de la Mano ${ }^{33-35}$ se han publicado en las revistas de mayor índice o factor de impacto (FI) como el JAMA $(\mathrm{FI}=45,217)$, The Lancet $(\mathrm{FI}=35,289)$, y BMJ $(F I=17,4)$. Recientemente saltaron todas las alarmas cuando Thomson Reuters presentaba los índices de impacto para 2014 de las revistas científicas. Por primera vez «el gigante americano» no seguía siendo el coloso de la investigación en el mundo, teniendo que dejar espacio a la publicación científica emergente de Europa y Asia (http:// ip-science.thomsonreuters.com/citationimpactcenter/). El mismo fenómeno se repetía a nivel de la historia de la Cirugía de la Mano en ese año; la revista oficial de Cirugía de la Mano europea The Journal of Hand Surgery European (JHSE) superaba por primera vez a la famosa versión americana The Journal Hand Surgery American (JHSA) en índice de impacto. El FI del JHSE consiguió por primera vez ser superior a 2.

Nuestra revista RICMA ha entrado por primera vez en la plataforma ELSEVIER y un nuevo futuro se abre para la revista oficial de la SECMA. Siempre habrá errores en la presentación y formato de un trabajo, incluso una vez publicado $^{36}$. Escribir un artículo original es una labor que se debe llevar de forma meticulosa, con sencillez y claridad.

En el presente artículo hemos querido exponer los puntos más importantes de la escritura científica y que se resumen en un ejemplo (tabla 1) de un trabajo ya publicado en el BMJ por uno de los autores del presente trabajo ${ }^{35}$. Esperamos que este trabajo ayude a los futuros autores de la REVISTA IBEROAMERICANA DE CIRUGÍA DE LA MANO a mejorar la calidad de las publicaciones de nuestra revista.

\section{Conflicto de intereses}

Los autores declaran no tener ningún conflicto de intereses.

\section{Agradecimientos}

Los autores quieren presentar el más profundo agradecimiento y dedicar este artículo al Prof. Dr. Manel Llusá y al Dr. José Antonio Medina, miembros de la SECMA, por el reconocimiento público al trabajo desarrollado por nuestro grupo de investigación expuesto durante la última reunión de carácter científico celebrada en la Universidad de La Laguna, Tenerife, el 22 de enero del 2016, y que se resume en este artículo. Gracias.

\section{Bibliografía}

1. Kronick D. A history of scientific and technical periodicals: The origins and development of the scientific and technical press. 2nd ed. Metuchen, NJ: Scarecrow; 1976.

2. Atkinson D. Scientific discourse in sociohistorical context: The philosophical transactions of the Royal Society of London, 1675 1975. Mahwah, NJ: Lawrence Erlbaum; 1999.

3. Day RA. How to write \& publish a scientific paper. 5th ed. Phoenix, AZ: Oryx; 1998. 
4. Atkinson $\mathrm{D}$. The evolution of medical research writing from 1735 to 1985: The case of the Edinburgh Medical Journal. Appl Linguist. 1992;13:337-74.

5. Huth EJ. Structured abstracts for papers reporting clinical trials. Ann Internal Med. 1987;106:626-7.

6. Sollaci LB, Pereira MG. The introduction, methods, results, and discussion (IMRAD) structure: A fifty-year survey. J Med Libr Assoc. 2004;92:364-7.

7. Kotsis SV, Chung KC. A guide for writing in the scientific forum. Plast Reconstr Surg. 2010;126:1763-71.

8. Franzblau LE, Kotsis SV, Chung KC. Scientific writing for enjoyable reading: How to incorporate style into scientific manuscripts. Plast Reconstr Surg. 2012;129:543-5.

9. Kotsis SV, Chung KC. Manuscript rejection: How to submit a revision and tips on being a good peer reviewer. Plast Reconstr Surg. 2014;133:958-64.

10. Manske PR. Structure and format of peer-reviewed scientific manuscripts. J Hand Surg Am. 2006;31:1051-5.

11. McIntyre E, Eckermann SL, Keane M, Magarey A, Roeger L. Publishing in peer review journals - criteria for success. Aust Fam Physician. 2007;36:561-2.

12. Rosales RS. Clinical research in hand surgery. J Hand Surg Eur. 2015;40:546-8.

13. Rosales RS, Rodriguez Pulido F, Atroshi I, Martin Hidalgo Y, McCabe SJ. Metodología para el desarrollo de un trabajo de investigación clínica en cirugía de la mano. Rev Iberoam Cir Mano. 2008;2:125-55.

14. Martin Hidalgo Y. Instrumentos PRO y niveles de evidencia en cirugía de la mano. Tesis Doctoral, Universidad La Laguna, Tenerife. 2016.

15. García Gutierrez R. La versión española del instrumento PRWE. Fiabilidad, validez y respuestas para valorar resultados en fracturas distales de radio. Tesis Doctoral, Universidad de La Laguna, Tenerife. 2016.

16. Hoogenboom BJ, Manske RC. How to write a scientific article. Int J Sports Phys Ther. 2012;7:512-7.

17. Rosales RS, Reboso-Morales L, Martin-Hidalgo Y, Diez de la Lastra-Bosch I. Level of evidence in hand surgery. BMC Res Notes. 2012;5:665.

18. Sánchez Rosales R, Hernández González VJ, Pollwein ME, Martín Hidalgo Y, Reboso-Morales L. Nivel de evidencia de la RICMA comparada con el JHSE y JHSA. Rev Iberoam Cir Mano. 2014;42:127-32.

19. McCabe SJ, Uebele AL, Pihur V, Rosales RS, Atroshi I. Epidemiologic associations of carpal tunnel syndrome and sleep position: Is there a case for causation? Hand (N Y). 2007;2:127-34.

20. Brogren E, Wagner P, Petraneck M, Atroshi I. Distal radius malunion increases risk of persistent disability 2 years after fracture. A prospective cohort study. Clin Orthop Relat Res. 2013;471:1691-7.

21. Gummenson C, Atroshi I, Ekdahl C. The quality of reporting and outcome measures in randomized clinical trials related to upper-extremity disorders. J Hand Surg Am. 2004;29: 727-34.

22. Montes L, Goyeneche J, Torre I, Rosales RS. Resultados del tratamiento de la artrosis carpo-metacarpiana del pulgar mediante implante Arex comparado con la artroplastia de Zancolli. Ensayo clínico aleatorizado. Resultados preliminares. Rev Iberoam Cir Mano. 2011;39:32-40.

23. Liberati A, Altman DG, Tetzlaff J, Mulrow C, Gotzsche PC, Ioannidis JP, et al. The PRISMA statement for reporting systematic reviews and meta-analyses of studies that evaluate health care interventions: Explanation and elaboration. Ann Intern Med. 2009;151:W65-94.

24. Tadjerbashi K, Rosales RS, Atroshi I. Intervention randomized controlled trials involving wrist and shoulder arthroscopy: A systematic review. BMC Musculoskelet Disord. 2014;25:252.

25. Sauerland S, Lefering R, Bayer-Sandow T, Brüser P, Neugebauer EA. Fingers, hands or patients? The concept of independent observations. J Hand Surg Br. 2003;28:102-5.

26. Alexandrov AV. How to write a research paper. Cerebrovasc Dis. 2004;18:135-8.

27. Chung KC, Kalliainen LK, Hayward RA. Type II. (beta) errors in the hand literature: The importance of power. J Hand Surg. 1998;23:20-5.

28. Alexandrov AV, Hennereci MG. Writing good abstracts. Cerebrovasc Dis. 2007;23:256-9.

29. Ward LG, Kedrach MG, Price SO. Accuracy of abstract for original research articles in pharmacy journals. Ann Pharmacother. 2004;38:1173-7.

30. Dias JJ, Rajan RA, Thompson JR. Which questionnaire is best? The reliability, validity and ease of use of the Patient Evaluation Measure, the Disabilities of the Arm, Shoulder and Hand and the Michigan Hand Outcome Measure. J Hand Surg Eur. 2008;33:9-17.

31. Rosales RS, Diez de la Lastra I, McCabe S, Ortega Martinez JI, Hidalgo YM. The relative responsiveness and construct validity of the Spanish version of the DASH instrument for outcomes assessment in open carpal tunnel release. J Hand Surg Eur. 2009;34:72-5.

32. Letchford A, Moat HS, Preis T. The advantage of short paper titles. R Soc Open Sci. 2015;2:150266.

33. Atroshi I, Gummesson C, Johnsson R, Ornstein E, Ranstam J, Rosén I. Prevalence of carpal tunnel syndrome in a general population. JAMA. 1999;282:153-8.

34. Atroshi I, Gummesson C. Non-surgical treatment in carpal tunnel syndrome. Lancet. 2009;374:1042-4.

35. Atroshi I, Larsson GU, Ornstein E, Hofer M, Johnsson R, Ranstam J. Outcomes of endoscopic surgery compared with open surgery for carpal tunnel syndrome among employed patients: Randomised controlled trial. BMJ. 2006;332:1473.

36. Bain BJ, Littlewood TJ, Szydlo RM. The finer points of writing and refereeing scientific articles. $\mathrm{Br} \mathrm{J}$ Haematol. 2016;172:350-9. 